\title{
X-SEGREGATION AND HETEROCHROMASY IN THE SPIDER ARANEA REAUMURI
}

\author{
KLAUS PÄTAU \\ Forschungsstelle für Biologie, Berlin-Dahlem, and \\ Johin Innes Horticultural Institution, Merton
}

Received 20.x.47

\section{INTRODUCTION}

THE spiders are distinguished by their sex chromosome mechanism. Painter (1914, partially in conformation of earlier work by Wallace and others) has definitely shown that the males of 13 species, each from another family, have two X's which pass to the same pole in the first meiotic division, thus leading to two kinds of sperms with either two X's or none. Hard (1939) described another case of this type. Only recently an exception was discovered by Revell (1947) in Tegenaria. He found the usual two X's in one species but three X's in two other species.

In all these cases the female must have twice the number of X's. This was first confirmed by Revell. But a comparative study of meiosis in males and females had never been undertaken. The main cytological problem in spiders-by what means the joint segregation of the X's is secured-was solved by Revell in Tegenaria, but it had yet to be seen whether the special mechanism in this genus is present in all spiders. In fact, it is not, so this question was still open. The material at hand, males and females, proved favourable not only to deal with these tasks but to gain some information concerning more general problems such as heterochromasy, nucleic acid metabolism and spiralisation. Furthermore, the question of the origin of the multiple $\mathrm{X}$-mechanism had to be raised and that cannot be treated adequately without genetical considerations which have been rather neglected so far.

The present study was begun in 1934 at the Kaiser Wilhelm Institut für Biologie in Dahlem but fortunately not finished, since, when this was done in Merton, the conditions in Tegenaria could be taken in full account. I am obliged to Dr Revell for much information on this genus and for letting me see his paper before it went to press.

\section{MATERIAL AND METHODS}

This study rests upon four individuals which were caught in a forest near Berlin in early August 1934. I am indebted to Dr Blumenthal for their identification. They were: 2 immature 
males with their last moult before them, I immature and I mature female.

The gonads were always removed from the abdomen before fixation. Because of the limited material only Bouin-Allen's fluid was used, which proved very satisfactory in every respect. From one ovary of the immature female a smear preparation with Feulgen staining was made. The other ovary, the eggs of the mature female and the testes were imbedded in paraffin wax after passing through methylbenzoat-celloidine. The thickness of the sections was either Io $\mu$ with Heidenhain's iron hæmatoxylin staining or 20 to $30 \mu$ with Feulgen staining. The Feulgen slides have faded but still show every detail.

All conclusions are based on nuclei clearly distant from the surfaces of the section, so misinterpretations owing to dislocations are excluded. The figures, if not mentioned otherwise, were made from sections. I am obliged to $\mathrm{Mr} \mathrm{La}$ Cour for his help with the photographs of plate (figs. 2-6).

\section{THE CHROMOSOME COMPLEMENT}

Table I shows that the diploid number is $2 \times 1 \mathrm{I}$ autosomes with $2 \mathrm{X}$ 's in the male and $4 \mathrm{X}$ 's in the female. Half of the sperms have $2 \mathrm{X}$ 's, the others none. The mature eggs have $2 \mathrm{X}$ 's.

TABLE I

Chromosome counts (with references to text-figs.)

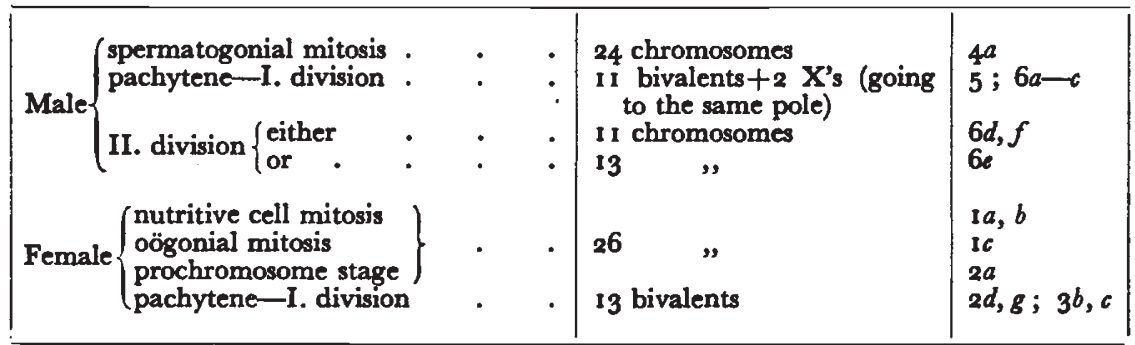

The X's cannot be distinguished from the autosomes except in spermatogenesis. Nor is it possible to identify any of the autosomes. All chromosomes are practically telocentric, the short arms being too small to be recognised.

\section{OÖGENESIS}

An excessive contraction of pre-meiotic chromosomes has been observed in the spermatogonial mitoses of several organisms (e.g. Chorthippus; Darlington, 1936). In Aranea this phenomenon does not occur in the male, at least not in the last spermatogonial division (fig. 4a), but it is extremely pronounced in all observed oögonial 
mitoses (fig. Ic) some or all of which must represent the last premeiotic division.
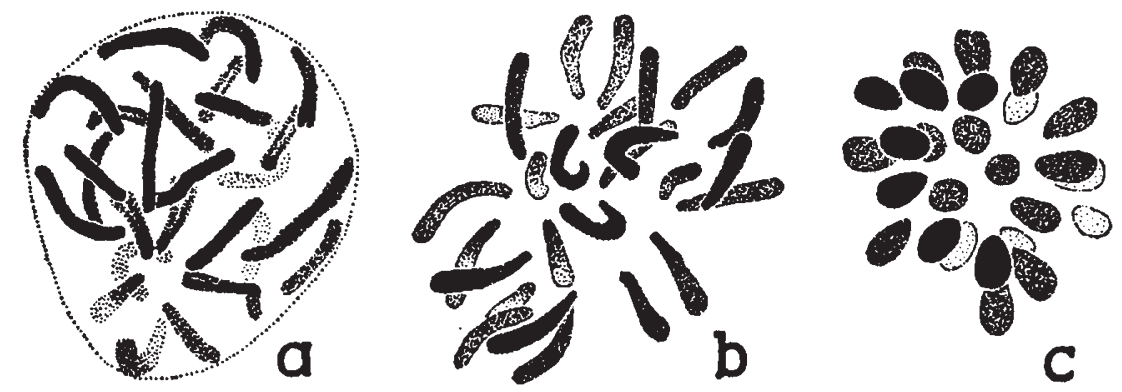

FIG. I.- $(a),(b)$ prophase and metaphase in cells of the stalk which carries the growing egg (in the text referred to as nutritive cells; corresponding anaphase in plate, fig. I);

(c) oögonial metaphase. Ovary of immature female. Feulgen. $\times 4000$.

A continuous seriation was possible beginning with the prochromosome stage (fig. 2a). These densely stained, irregular bodies are complete chromosomes: they have nothing to do with heterochromasy which has not been observed at all in the female. The prochromosomes unravel into leptotene chromosomes (fig. $2 b$ and $c$ ). Both in leptotene and in pachytene polarisation seems to be missing. But the number of nuclei was too small to be quite sure about that. A moderate degree of polarisation in the leptotene of Aranea could be recognised only in favourably orientated nuclei since a full analysis of the crowded chromosomes is impossible. In the pachytene nucleus of fig. $2 d$ the polarisation might have already lapsed. In this and in another pachytene nucleus (fig. $2 e$ ) one chromosome was only partially paired, possibly owing to structural hybridity. Chromomere structure could be seen only exceptionally (fig. $2 f$ ).

In diplotene the following changes begin and proceed quickly : (i) The thymonucleic acid charge, as judged by the Feulgen staining, decreases, and soon after the stage of fig. $2 h$ it seems to disappear completely. In eggs, like that of plate, fig. I, not the slightest staining of any part of the cell can be seen in spite of the thickness of the section $(30 \mu)$. As long as the chromosomes are visible no change of their size can be ascertained. (ii) A rapidly growing nucleolus appears between the stages of figs. $2 g$ and $2 h$. It develops an outer layer of optically denser material (plate, fig. I). (iii) An enormous growth of the nucleus and cytoplasm takes place. The inter-relation between these three changes need not be discussed here as the most important ribose nucleic acid metabolism could not be followed with the methods applied.

The mature female had obviously not yet copulated for no sperms were found and no oviposition occurred, although she was kept for one-and-a-half months before killing. Only first metaphases (5) and anaphases (27) were observed (fig. 3). The 13 bivalents have exactly 
one chiasma each. Most are terminalised but the crowding makes it impossible to determine the terminalisation coefficient accurately. It is obviously higher than in the male.
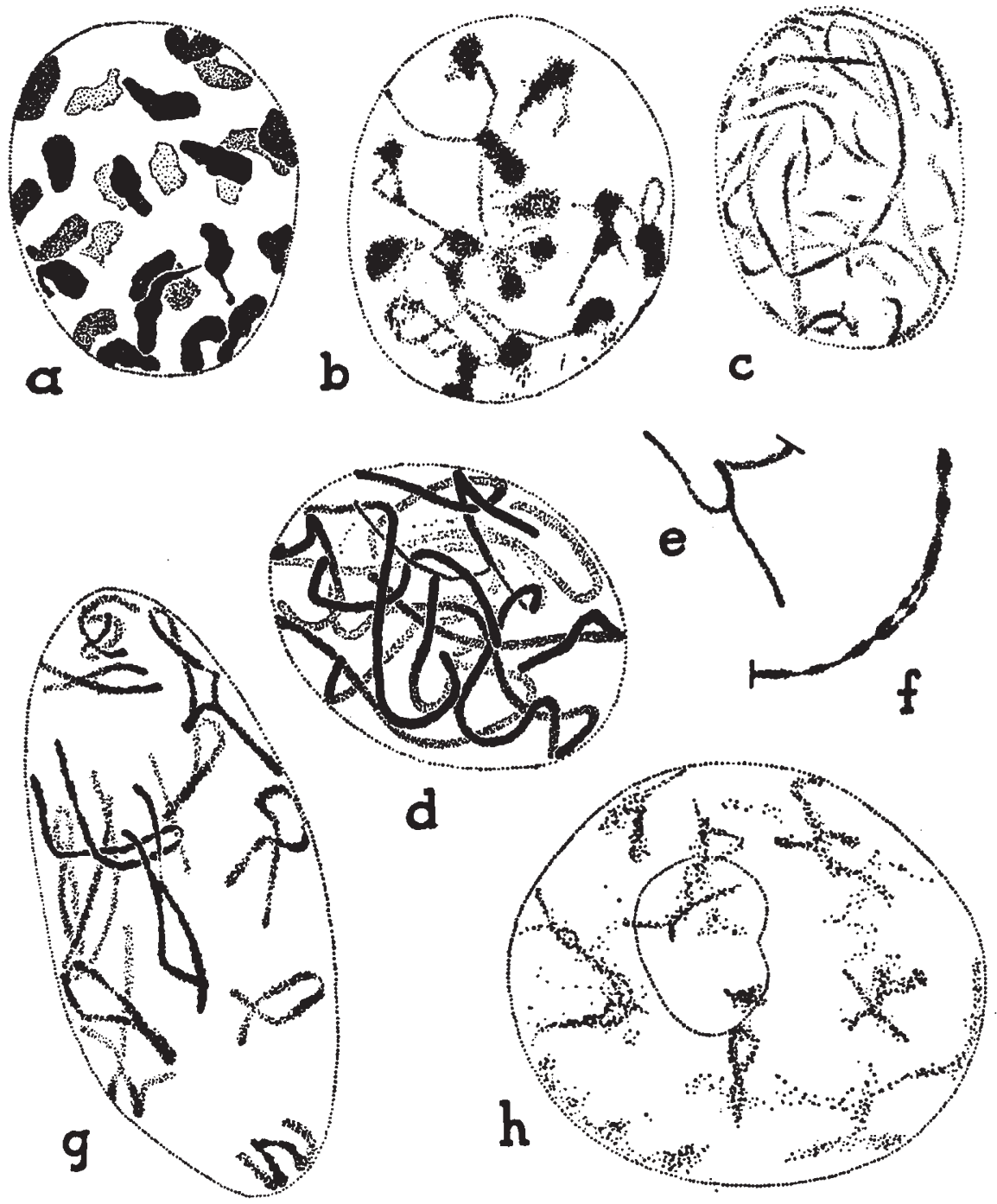

Fro. 2.-Oöcyte nuclei from immature female : (a) prochromosomes ; $(b)$ unravelling of prochromosomes (only some of them drawn); (c) leptotene (optical section); (d) pachytene; (e) same, incomplete pairing; $(f)$ same, chromomere structure ; $(g),(h)$ diplotene (oblong shape of nucleus $g$ probably due to smearing); $(b),(f)$, (g) smear. Feulgen. $\times 4000$.

The gaps which have been left in Aranea by this description are filled by Montgomery's paper on the oögenesis and fertilisation of the egg of Theridium ( 1907 ). It begins with the appearance of diplotene chromosomes after the growth period. They have then 
more than ten times the length they will have in first anaphase. In view of my observation of relatively short chromosomes in early diplotene (fig. $2 g$ ) the conjecture seems justified that the invisible chromosomes of the later growth period are considerably longer than in early diplotene.
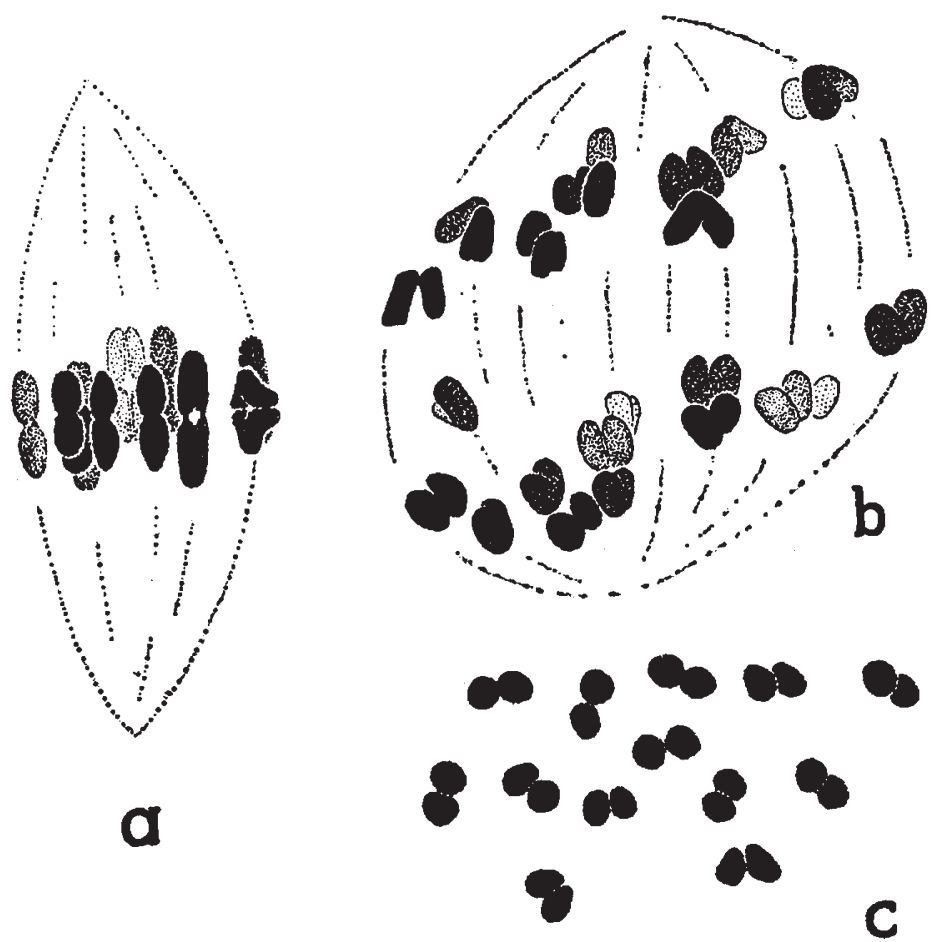

Frg. 3. First meiotic division in the egg. (a) metaphase (not all chromosomes visible); $(b)$ anaphase ; (c) same, polar view. Feulgen. $\times 4000$.

Montgomery's figures show absence of conspicuous heterochromasy not only in the egg nucleus but also in the male pronucleus. It must be concluded that the sperm X's like those of the female pronucleus do not behave heterochromatically in the fertilised egg.

\section{SPERMATOGENESIS (AUTOSOMES)}

From the prochromosome stage until early diplotene, when the egg enters the growth period, the behaviour of the autosomes is very much the same in both sexes, except that polarisation may be missing in the female. In the male it has been observed from leptotene to early pachytene (figs. $4 c$ and $5 a$ and $b$ ). The general course of events can be seen in figs. $4-6$ and in plate, figs. $2-6$. The cells are included in cysts, each of which contains 16 spermatocytes of the first order or corresponding numbers of other stages. Usually all cells 
within one cyst develop strictly simultaneously and without abnormalities.

The metaphase bivalents have always exactly one chiasma which is situated in the long arm. Apparent ring bivalents in diplotene (fig. $5 h$ ) might be interpreted as having also only one true chiasma whilst the short arms are merely sticking together, as it has never been proved that a real ring bivalent can be opened before anaphase. But it has not been disproved either. Occasional bivalents like that of fig. $5 g$ seem to suggest that the short arms may form a chiasma which, however, being too close to the centromere, cannot prevent these arms becoming separated before metaphase. The terminalisation coefficient in metaphase varies little around 5/II-6/I I and seems not to be correlated with the highly variable chromosome length which will be discussed later.

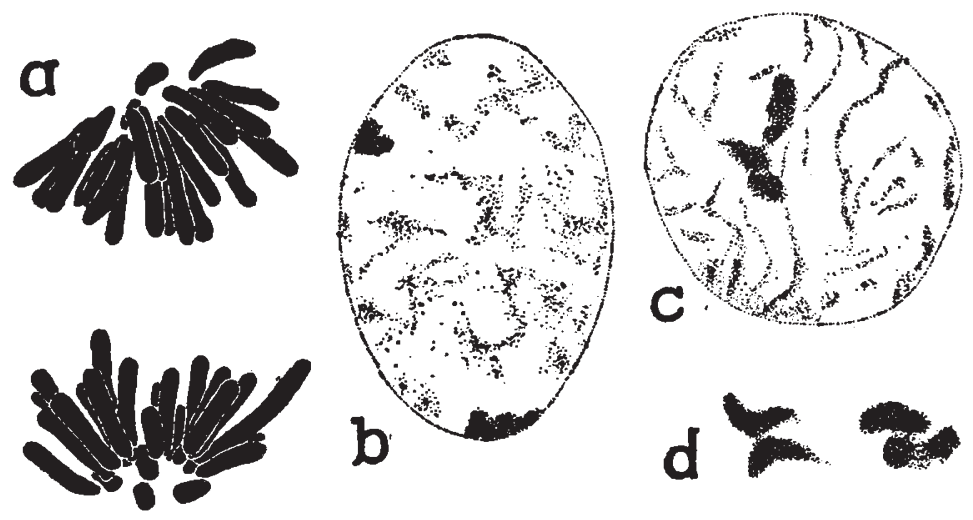

FIG. 4.-(a) last spermatogonial division (from cyst with 8 divisions); (b) unravelling of prochromosomes (optical section); (c) leptotene (X group from upper surface, otherwise optical section); (d) X group from leptotene nuclei. Feulgen. $\times 4000$.

Failure of chiasma formation, as indicated by a pair of univalents, occurred rarely. In fig. $7 a$ this was clearly due to abnormal conditions in the whole cyst, as the cyst contained all sorts of stages between late diplotene and early spermatids and as the chromosomes in these nuclei made a somewhat swollen impression.

A few inversion bridges and fragments were observed in both males (fig. $7 b$ and $c$ ). In one case the fragment could not be found (fig. $7 d$ ), possibly it was hidden amongst other chromosomes. The size of the fragments seems to vary. A very careful search did not give any indication of a pachytene loop, though at least in the nuclei $5(a$ and $b)$ a complete analysis was possible ; all chromosomes were paired in full length.

The diplotene nucleus of fig. $5^{e}$ shows an unusual translocation which involves only single chromatids. The diagram (fig. $5 f$ ) is arbitrary in details but the main facts are clear. Two breaks (one distal and one proximal, both in the long arms of two non-homologous 
chromosomes) have presumably taken place in the preceding resting stage. After the division, the original connection must have been

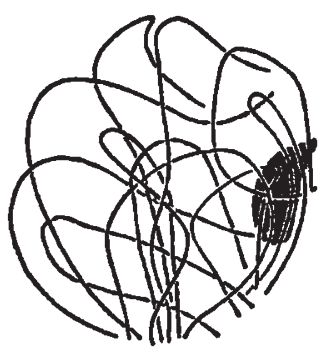

a

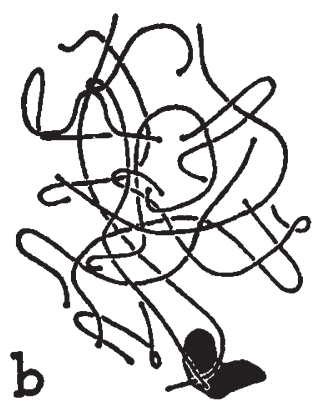

c

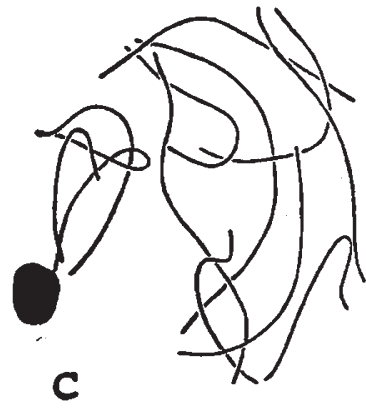

$S(1)$

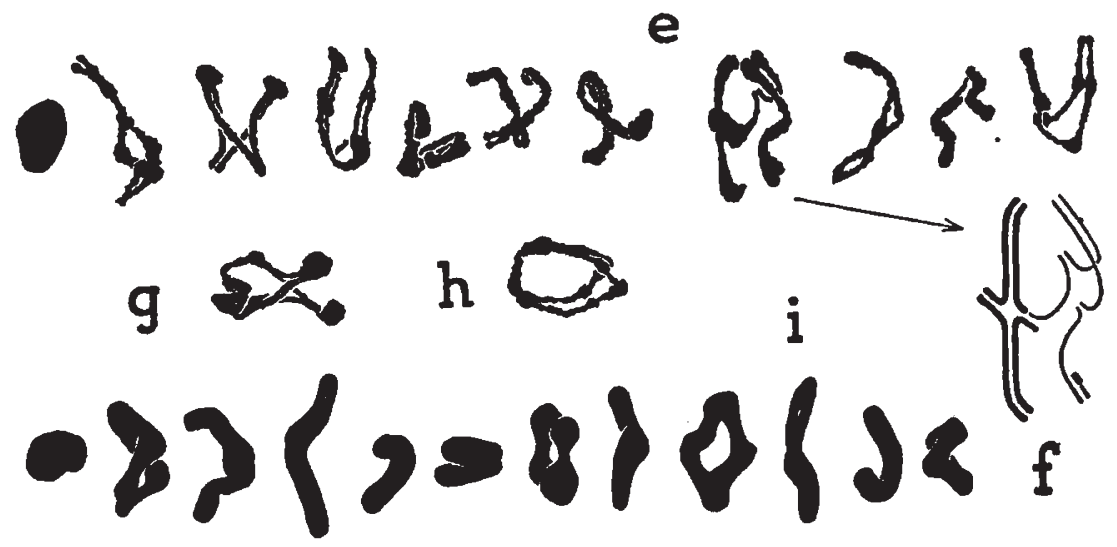

Fic. 5.-Spermatogenesis. (a), (b) early pachytene; (c) late pachytene; $(d)$ early diplotene; $(e)$ middle diplotene ; $(f)$ diagram of chromatid translocation in (e) ; $(g)$, (h) same, bivalents with possibly two chiasmata ; (i) diakinesis. (g) ironhæmatoxylin; $(a-f),(h),(i)$ : Feulgen. $\times 4000$.

restored at both breaks in only one chromatid, thus leaving the other chromatid liable to translocation.

\section{COMPARISON WITH TEGENARIA}

In view of the peculiarities discovered by Revell (1947) in the spermatogenesis of Tegenaria I carefully re-examined my slides. Aranea Reaumuri differed from Tegenaria in the following features :-

(i) The X's are less polarised than the autosomes. In pachytene the $\mathrm{X}$ group is lying on the periphery of the field of polarisation 

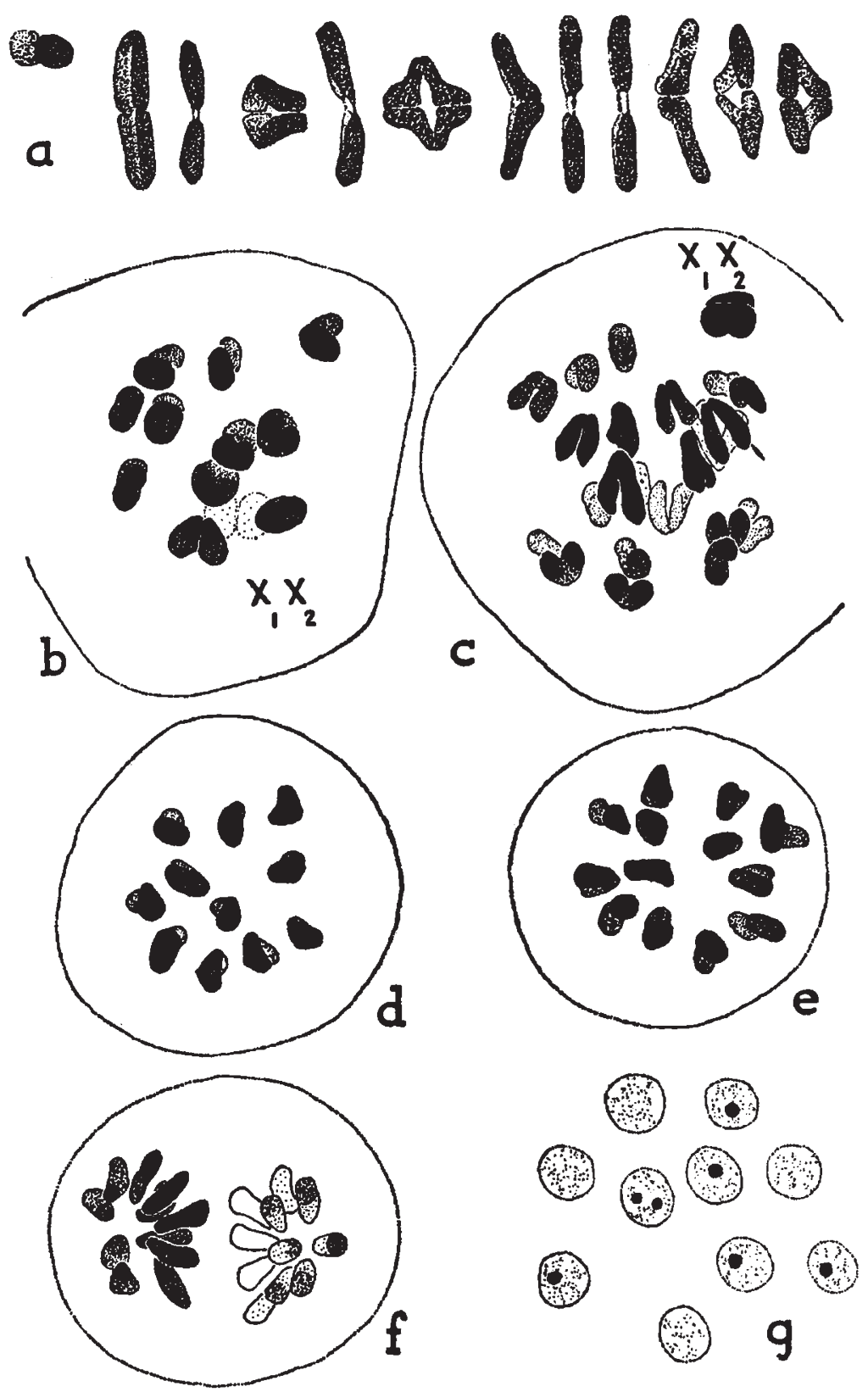

Fro. 6.-Spermatogenesis. (a) I. metaphase (X's in end view, their real position was nearer to the pole); (b) same, polar view; (c) I. anaphase ; $(d),(e)$ II. metaphase without and with X's; $(f)$ II. anaphase; $(g)$ young spermatid nuclei with and without heterochromatic X's. Feulgen. $(a-f) \times 4000 ;(g) \times 1300$. 
(fig. $5 a$ and $b$ ). (ii) Most pachytene autosomes are polarised at both ends (fig. 5a). (iii) Polarisation lapses at the end of pachytene. In diplotene (plate, figs. 2 and 6) and diakinesis (plate, fig. 3) the bivalents are evenly distributed, no "stage resembling anaphase precedes metaphase" and the position of the X group is independent of that of the centrosomes (plate, fig. 2). (iv) The X's show strong positive heterochromasy until diakinesis; in first metaphase their

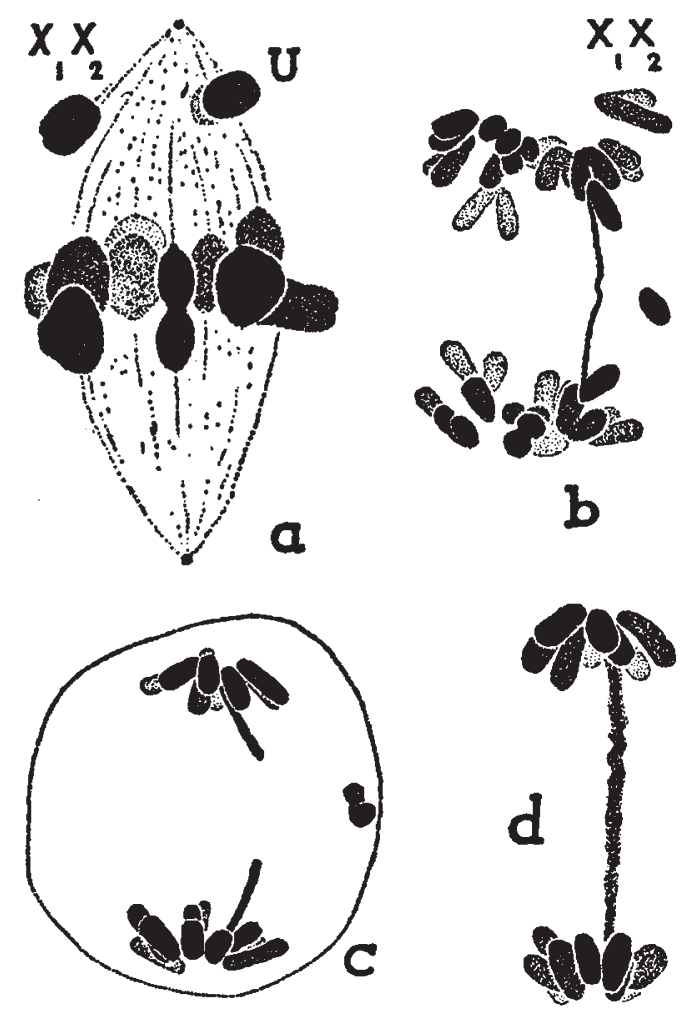

Fro. 7.-Spermatogenesis. (a) I. metaphase with two univalents (U) ; (b) I. anaphase with inversion bridge and fragment ; (c) II. anaphase with broken bridge and fragment ; (d) II. anaphase with bridge. (a), (d) iron-hæmatoxylin; (b), (c) Feulgen. $* \times 4000$.

nucleic acid charge cannot be much lower than that of the autosomes. (v) The ends of the pachytene autosomes are not heterochromatic.

The $\mathrm{X}_{1} \mathrm{X}_{2} \mathrm{O}$-mechanism is characteristic of all spiders so far investigated (with an additional $\mathrm{X}$ in two Tegenaria species), and in Tegenaria the lasting polarisation offers an explanation of why the $\mathrm{X}^{\prime}$ 's always pass to the same pole in the first division. One might therefore have thought that the unusual conditions in Tegenaria would be common to all spiders. It is quite possible that former students of the cytology of spiders merely failed to notice them. The normality of the Aranea spermatogenesis, however, suggests that the cytological 
peculiarities in Tegenaria are exceptional even with spiders. The primary cause for the regular segregation of the X's must be sought on other lines.

\section{SEX CHROMOSOMES}

\section{(i) Heterochromasy}

Whilst autosomal heterochromatic regions, if they exist in Aranea Reaumuri, must be very small, the X's seem to be totally heterochromatic. This property, it seems, becomes visible only in spermatocytes and spermatids. No differential behaviour of the $X$ 's and no chromocentres could be seen in oögenesis (fig. 2), in oögonial, spermatogonial or other mitotic prophases and resting stages of the gonads, or in any other tissue that happened to be included in the sections.

There is ample cytological and genetical evidence that euchromatin and heterochromatin differ in their permanent chromonema structure, generally assumed to be a protein framework forming a chain of chromomeres, genes, to which nucleic acid is attached in cyclically changing quantities. Under special physiological conditions, such as exist normally in certain cells or can be induced by artificial treatment, heterochromatin manifests itself by a differential appearance which is called heterochromasy or heteropycnosis. In order to simplify the terminology it may be suggested to retain only the first term which has also the advantage to indicate by its verbal meaning what seems to be the essential feature of the phenomenon : a nucleic acid charge different from that of euchromatin in the same nucleus. Darlington and La Cour (1940) have interpreted the facts by the assumption that heterochromatic genes have a lower nucleic acid reactivity which can upset the correlation, characteristic for the euchromatin, of nucleic acid attachment and reproductive cycle.

The nucleic acid charge and the chromosome thickness of heterochromatic segments deviate in the same direction from those of euchromatic ones, the convenient terms positive and negative heterochromasy are therefore synonymous with positive and negative heteropycnosis (White, 1935).

The existence of heterochromatin should not be taken for granted if the heterochromasy is displayed by chromosome sections which might have a chemical environment different from that of other regions. A conclusive example has been provided by Caspersson and Schultz (1938). They showed that the nucleic acid cycle of euchromatic genes is disturbed by translocation to heterochromatic segments. Another spatial relation, proximity of the centromere, presumably determines the over-charge of proximal chromosome parts which occurs during the meiotic prophase in some organisms (Darlington and La Cour, 1940).

In the permanent telophase nuclei of certain Radiolariæ the distal regions of two exceptionally long chromosome arms are over-charged 
which are lying on the surface of the densely packed chromosome mass (Pätau, 1937a). It seems not unlikely that the excessive nucleic acid charge is caused merely by the position of these regions. The possibility that the heterochromasy of polarised chromosome ends in Tegenaria (Revell, 1947) is not due to heterochromatin but to contact with the nuclear membrane should be borne in mind.

In the X's of spiders and other organisms the existence of heterochromatin is, however, beyond doubt. Darlington (1937, pp. 31 2-3 I3) and White (1940a) have given several reasons why the heterochromasy of the $\mathrm{X}$ in spermatogenesis cannot be merely the result of their unpaired condition. Other convincing evidence is provided by the spermatids. In Aranea the X's are indistinguishable from the autosomes in the second division. Nevertheless they turn out extremely heterochromatic in the subsequent stages. Since the genetical balance in the X-spermatids is the same as in female cells the heterochromasy must be determined by physiological conditions which are not immediately controlled by the genetical constitution of their own nucleus.

Selection will take care that the normal physiological conditions in the oöcytes are such as to prevent a condensation of the X's which could interfere with chiasma formation and consequently upset the regular X-disjunction. (For a discussion in terms of the precocity hypothesis, see Darlington, 1939.) The adaptive value of the absence of heterochromasy in the female meiosis has already been pointed out by White (1940a).

\section{(ii) Heterochromasy and spiralisation}

In the female the X's could not be identified. All stages, including pachytene, showed 13 or 26 chromosomes of equal appearance and with length differences comparable to those observed in the male. Therefore, with equal nucleic acid charge, heterochromatin and euchromatin must have the same or nearly the same properties in regard to pairing, division, chiasma formation and spiralisation.

Darlington and La Cour (1938, 1940) have produced negative heterochromasy by cold treatment in Paris and in Trillium. The heterochromatic segments retained their normal length but were considerably reduced in diameter. Constant length, independent of the thickness, was also observed by White (1940a). .He found in several Orthoptera that the totally heterochromatic $\mathrm{X}$ in mitotic and meiotic metaphases and in diakinesis had the same length with a varying diameter.

The fact that heterochromatic segments, when short of nucleic acid, may have only half their normal thickness can hardly be understood without assuming some change in the spiral structure. Otherwise the spiral could normally fill only a central core, being covered by a thick layer of nucleic-acid-containing material. I regard that as 
incompatible with all reliable observations (this will be discussed elsewhere). The diameter of the spiral itself must vary with the nucleic acid charge. Nevertheless the length of the spiral remains the same.

The characteristics of a spiral are the height $h$ of one gyre, the diameter $d$ of the spiral and the length $c$ of the coiled thread, the chromonema, to whose axis they are all related (fig. 8). The axis we may assume to be the protein framework to which the nucleic acid is attached.

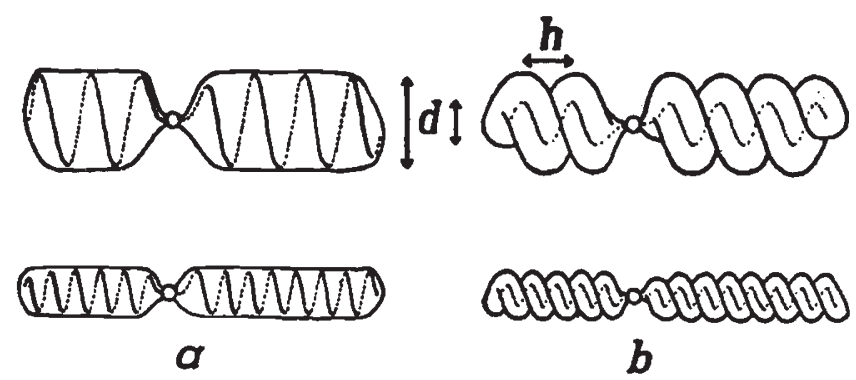

Fro. 8.-Explanation in the text.

If $c$ as well as $d$ varied with the nucleic acid charge, $h$ would have to follow a certain non-linear function of $c$ and $d$ in order to render the chromosome length $l$ constant. It is very difficult to imagine a mechanism which would secure this function. Let us, therefore, assume that $l$ and $c$ are both constant. Then the proportion $h: d$, whatever its value be, must be constant too. This again would be very difficult to account for if a thin chromonema were imbedded in a homogeneous matrix (fig. 8a). The whole difficulty disappears if most of the chromosome material is attached to the chromonema and if this thick thread is coiled into a compact spiral (fig. 8b). The length of a compact spiral is independent of the thickness of the thread. If the chromonema is cylindrical $l$ is always equal to $c 2 \cdot 72$.

Thus the otherwise quite enigmatical independence of length and diameter of a heterochromatic segment can be fully understood from three very plausible assumptions : ( $\mathrm{I}$ ) The metaphase spiral is formed by a thick thread with no space left between succeeding gyres. This view, because it most easily explains the regularity of the spiral structure, is held by many cytologists, including Darlington and Upcott, who have discussed it in detail (1939). (2) The metaphase spiral is compact. That is a reasonable assumption as the compact condition, distinguished by a maximal degree of contact within the spiral, is likely to be the most stable one. It has already been given prominence by Darlington and others. (3) The chromonema length in metaphase has a definite value independent of the nucleic acid charge which determines only the chromatid diameter. 
Fig. I.-Growing egg from immature female (nucleus a little out of focus). Feulgen. Taken with narrow aperture. $\times 45^{\circ}$.

Fig. 3.-Diakinesis in male with centrosomes opposite to each other. Iron-hæmatoxylin. $\times 2400$.

FIG. 4.-I. metaphase with exceptionally separated X's. Iron-hæmatoxylin. $\times 2400$.

Fig. 5--Typical I. metaphase. Ironhæmatoxylin. $\times 2400$.

Fro. 6.-Testis. Two o'clock : early diplotene; $\mathrm{X}_{d}: \mathrm{X}$ group at late diplotene ; $l$ : I. metaphase with long chromosomes (X group in typical position); $s:$ same with short chromosomes (X group out of focus); $\mathrm{X}_{m}$ : $\mathrm{X}$ 's at I. metaphase ; $\mathrm{X}_{8}$ : $\mathrm{X}$ chromocentre in young spermatid; five o'clock: old spermatids. Feulgen. $\times$ I 200 . 

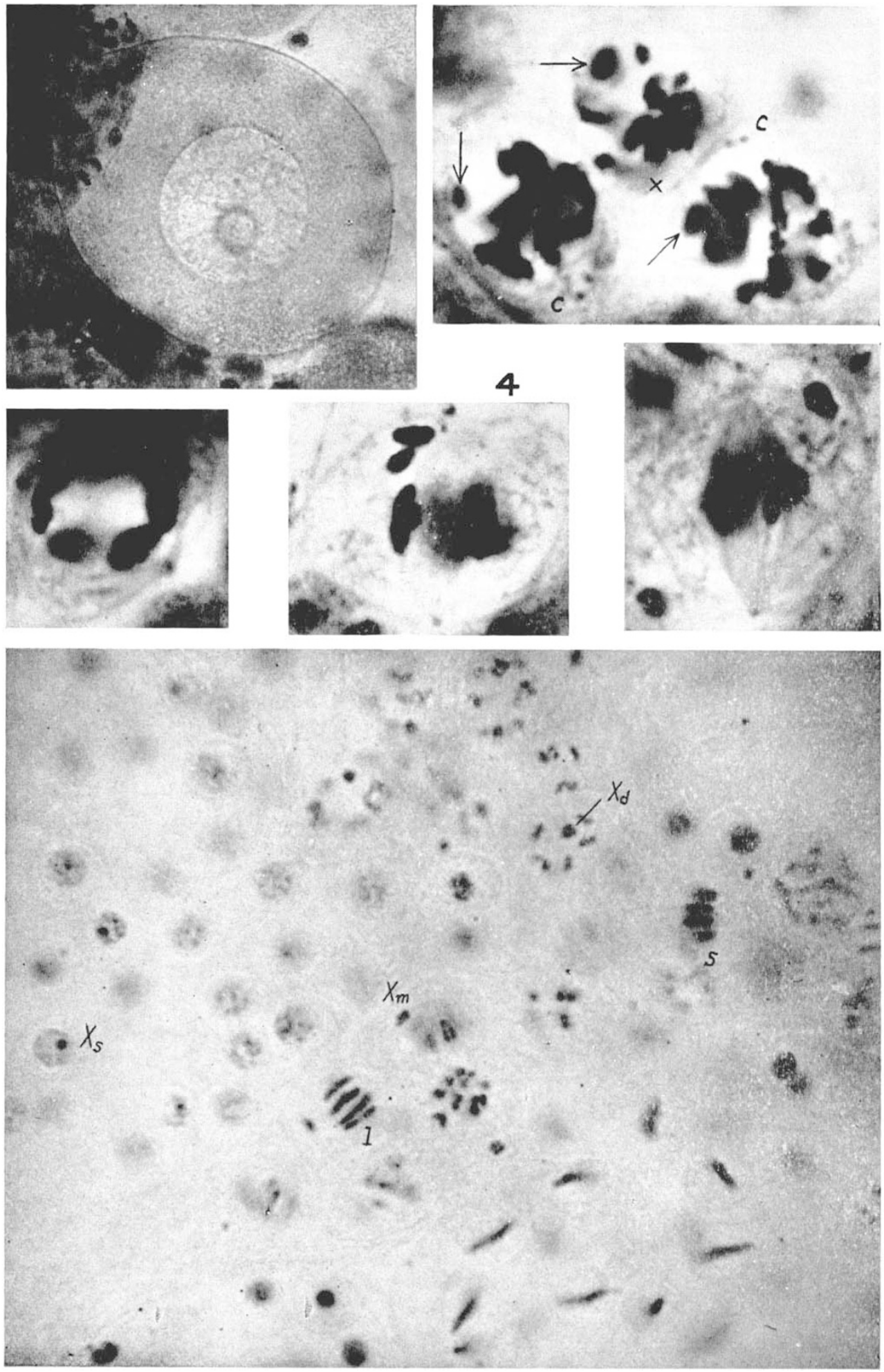
A real independence, within certain limits, between chromatid length and diameter would be new evidence, not easily to be dismissed, in favour of these assumptions. The question is whether the existing observations do prove independence. It could, of course, happen that changes of different spiral characteristics by pure chance approximately cancel one another in their influence on the chromosome length. But if length constancy is found in quite different material this possibility becomes remote. So the results of Darlington and $\mathrm{La}$ Cour and White are probably significant.

The suggested explanation of the length constancy implies that the number of coils per length unit is inversely proportional to the chromatid diameter. White's (1940a) observations of relic coils in spermatogonial prophases of three Acrididæ seem indeed to indicate a relatively higher number of coils in the negatively heterochromatic $\mathrm{X}$ than in the autosomes. White's view on spiral structure in eu- and heterochromatin is essentially the same as the one taken here, though he has apparently not realised that a compact structure appears to be the only available explanation of the length constancy. He even throws some doubt on the compactness of negatively heterochromatic chromosomes because " their outlines are too irregular and flocculent." But I think this outline is quite in accordance with the expectation (fig. $8 b$ ) ; the question is rather why the fully charged metaphase chromosomes show a smooth outline. In big mitotic chromosomes the furrows between the coils should be easily visible even in living cells, but this is usually not the case. It is not unlikely that some of the nucleic acid containing chromosome material is not fixed to the chromonema but fills these furrows.

The length constancy is certainly not unconditional. In cases of extreme nucleic acid starvation, heterochromatic segments have been observed to be longer as though in a prophase condition (e.g. in Paris japonica: Darlington and La Cour, 1940). If the metaphase chromatid really is a compact spiral with its small shortening factor (as we may call the proportion chromonema length : chromatid length) of $2 \cdot 72$, the prophase contraction must be due to a shortening of the chromonema itself in addition to spiralisation. Perhaps this shortening is somehow connected with the attachment of nucleic acid. Further nucleic acid accumulation would increase only the diameter of the chromonema. If its initial contraction proceeded along slightly screwed lines on the chromonema it would provide the inner cause, the molecular coiling of Darlington, for the exterior coiling.

Another approach to the problem of the relation between nucleic acid charge and spiralisation is offered where, as in meiosis in the male of Aranea Reaumuri, the heterochromatic regions are more contracted in prophase than in metaphase. Their heterochromasy begins when they, as well as the autosomes, are in a prochromosome condition; it becomes pronounced when the autosomes unravel and 
quickly change into leptotene. During this transformation the X's retain their shape, though their nucleic acid charge may already have begun to increase. A comparison between figs. $4 c$ and $2 a$ shows that the structure of the X's in early leptotene of the male must be the same as that of all chromosomes in the prochromosome stage of the female (or of the male). The outline of these chromosomes and especially the way they unravel (fig. $2 b$ ) leave no doubt that they are not in a state of regular coiling. They behave as if the chromonemata were sticky and as if subsequently their stickiness ceased or were overcome by a repulsion. During this process the nucleic acid charge seems to decrease.

In the male the X's do not unravel. Their nucleic acid content increases greatly until in diplotene they have condensed into one round body (fig. 5). Later their positive heterochromasy disappears as the nucleic acid charge of the autosomes is enhanced. Possibly at that time the $\mathrm{X}$ group loses some of its nucleic acid. In metaphase each $\mathrm{X}$ appears to have about the same degree of condensation as the autosomes. A slightly negative heterochromasy might exist at metaphase, though that is difficult to ascertain as the $\mathrm{X}$ chromatids are still more closely paired and as the exact length proportion between the X's and the autosomes is unknown. In any case the spiral structure of the X's must be regular and not very different from that of the autosomes (plate, fig. 6 at $\mathrm{X}_{m}$ ). This is also indicated by the fact that the condensation of the X's and that of the autosomes show intercystic covariation, they are obviously controlled by the same physiological conditions within cysts. At least in the cysts with longer chromosomes the metaphase X's are longer than in late diplotene.

Now coiling is certainly a progressive process which cannot be reverted. Once the regularity of a spiral has been lost it will not be restored. Therefore the metaphase spiral of an X can hardly have derived from those irregular relic coils which might still have existed in the prochromosome. Neither does it seem at all likely that the longer metaphase spiral has developed from a preceding shorter spiral as this would involve a decrease in diameter and an increase in the number of coils. The chromonema must undergo a new process of coiling within the relatively over-charged $\mathrm{X}$ as it does in the stretched and under-charged autosomes. Spiralisation is independent of excessive nucleic acid.

The undisturbed progress of coiling within the $\mathrm{X}$ would be hard to understand if the chromonema were kept together by a general attraction or by the stickiness which is possibly responsible for the preceding prochromosome condition. This difficulty may be taken as a new argument in favour of the assumption that positive heterochromasy consists in the accumulation of a nucleic acid containing fluid which finally runs into one drop. Within this the chromonema would be free to undergo spiralisation and the surface tension would result in the round shape and smooth outline of the chromosome. This 
fluid has been assumed by Darlington and La Cour (1945) to be unpolymerised nucleic acid distinguished from the polymerised nucleic acid building up the normal "dry" chromosome. They have discussed in detail the importance of the stickiness of this fluid in relation to chromosome breakage. Stickiness of heterochromatic chromosome sections has been observed in many organisms (e.g. in Edessa: Schrader, 194I) and it has recently been shown by Thomas and Revell (1946) in Cicer to be responsible for secondary association.

Stickiness can be displayed, of course, only if the well-known repulsion between chromosomes, whatever it be, is sufficiently weak. The apparent absence of stickiness in euchromatic chromosomes does therefore not prove that the fluid nucleic acid is absent too. On the contrary, we may assume that this fluid is the same which has been suggested above as reason for the smooth outline of the normal chromosome from late prophase to anaphase. It should be noted that this conception, though it abandons the restriction of unpolymerised nucleic acid to the heterochromatin, still fulfils all what is required by the breakage theory of Darlington and La Cour.

To avoid a new term the fluid may be called matrix, but it must be understood that it is based on the assumption of a compact metaphase spiral or possibly a hollow spiral (Darlington and Upcott, 1939), in which case the matrix would fill not only the furrows but also the inner core. There is little connection between this and other matrix hypotheses involving different types of spiral structure (e.g. fig. 8a), so we may refrain from discussing these hypotheses.

With increasing negative heterochromasy the matrix disappears first, then the (polymerised) nucleic acid is reduced which is attached to the chromonema and determines its thickness. Stickiness betrays the presence of a matrix, though it may be only a thin film, as possibly in the prochromosomes of Aranea, covering the chromonema. A positively heterochromatic segment has more matrix material than euchromatic regions in which it may be even completely missing at that stage. Certain Protista, for instance Merodinium (Pätau, 1937b), seem to have no matrix at all.

The attachment of a certain amount of (polymerised) nucleic acid is presumably necessary for spiralisation (Darlington, 1947) and, if we assume a compact metaphase spiral, for the shortening of the chromonema itself. Further accumulation, however, increases only the chromonema diameter and therefore (again assuming a compact spiral) must enlarge the thickness of the chromatid but not its length. The presence of a matrix does not influence the coiling, though it may, by its surface tension, add to the stability of a compact spiral or, with positive heterochromasy, draw together a prophase or resting stage chromosome in an irregular way, and thus produce extreme over-condensation. 
(iii) $X$-segregation in the male and heterochromatic stickiness

The $\mathrm{X}_{1} \mathrm{X}_{2} \mathrm{O}$ mechanism depends on joint segregation of the $\mathrm{X}$ 's. This is already secured in first metaphase by what seems to be a fibrous connection between the $\mathrm{X}$ group and one centrosome. This is apparently true in all spiders. The reason for the $\mathrm{X}$ 's never being connected with different centrosomes must be their close pairing from which no exception could be found in Aranea between pachytene and diakinesis. The special problem the cytologist finds himself confronted with by the spiders is : why do the X's pair? Under the erroneous impression that the X's were always of equal length it has been assumed that they pair because they are homologous, although the strong positive heterochromasy prevents chiasma formation. The following evidence will leave little doubt that their pairing is caused simply by heterochromatic stickiness :-

(I) The initial pairing can be observed during the pre-meiotic unravelling of the autosomes and in early leptotene. Besides many nuclei with yet unpaired X's and others with more or less complete pairing there are numerous nuclei in which the X's are connected only at single points which apparently do not correspond to one another but represent chance contacts resulting from a random chromosome movement within the nucleus (fig. $4 b-d$ ). If the X's were brought together by an attraction one should expect them to be drawn together quickly once they have met. Instead of that their relative movement is obviously nearly arrested by the first contact. The slow completion of the pairing until pachytene and the smooth outline of the strongly heterochromatic X's is presumably the result of matrix accumulation (fig. 5).

(2) The pairing of the X's in spiders seems always to start with the beginning of their positive heterochromasy. In Aranea the heterochromasy becomes clearly visible when the autosomal prochromosomes unravel. Then the pairing, as described above, is already on its way. But the heterochromasy certainly begins somewhat earlier, as it is difficult to ascertain whether two out of twenty-four irregular prochromosomes are slightly stronger stained.

In other spiders the heterochromasy arises much earlier and in these cases pairing takes place much earlier too. In Mavia vittata (Painter, I9I4) the X's remain condensed after the last spermatogonial division and their fusion is accomplished during the resting stage before prochromosomes are formed. Revell's observations in Tegenaria start with the prochromosome stage. Here again the $\mathrm{X}$ 's are already heavily heterochromatic and appear always as one round body with smooth outline, quite different from the shape of the X's in Aranea even in leptotene.

During the telophase of the second division the X's become heterochromatic and fuse into one spherical chromocentre (plate, fig. 6). In about ro per cent. of the young X-spermatids, however, the 
X's are still separated (fig. $6 g$ ), and this frequency does not appreciably diminish during the subsequent development, thus proving that very soon after the end of telophase the growing density of the nucleus, which becomes increasingly smaller, inhibits any further chromosome movement.

(3) With the end of positive heterochromasy the pairing relaxes. In Aranea and probably in most spiders the over-condensation of the $\mathrm{X}$ 's has disappeared in metaphase and their pairing seems to be but a residual of the preceding close fusion (figs. $6 a$ and $c, 7 b$; plate, fig. 6 , at $\mathrm{X}_{m}$ ). In one metaphase the $\mathrm{X}$ 's had even completely separated (plate, fig. 4).

In Tegenaria the positive heterochromasy of the X's disappears much earlier and is followed by negative heterochromasy in early leptotene. Consequently, so we may conclude, the $\mathrm{X}$ 's fall apart long before real metaphase has been reached, though they still retain a sort of distant pairing (Revell's plate, figs. 4, 5 and I 3 ). But this might be due to the obstruction of sideways movement by orientated spindle material. The early separation of the $X$ 's could easily upset their joint segregation if not a complementary mechanism, continuous polarisation, had been established which keeps both X's to the same centrosome. In the phylogenesis of Tegenaria continuous polarisation was probably the supposition without which selection would have prevented the disappearance of positive heterochromasy in early diakinesis. But even so heterochromatic stickiness might still be important in diplotene as an additional guarantee that both X's stay near the same (proximal) centrosome when the other centrosome starts moving around the nucleus.

During the first metaphase the still, if less closely, paired X's stay outside the spindle all the time (plate, fig. 5), which is apparently the case in all spiders. Whilst the distance between the X's and the centrosome is always small in Aranea Reaumuri, it can be large enough in other species to keep the X's (outside the spindle !) in the equatorial plane (Painter, I9I4). It seems that both $\mathrm{X}$ 's are attached to separate fibres connecting their centromeres with the same centrosome. The transgression to metaphase has not been followed, but the fact that the $\mathrm{X}$ group in this stage was always seen proximate to one centrosome can perhaps be best accounted for by the assumption that the fibrous connection between the X's and one centrosome is established when by random movement the $\mathrm{X}$ group has reached a position sufficiently near this centrosome. This distance might possibly be the length of already existing monocentric aster fibres. It is not necessary to assume that the chromosome-fibre system produces an active movement. By merely limiting the range of movement the X-centrosome connection might already guarantee the inclusion of the $\mathrm{X}$ 's in one telophase nucleus.

In cells with two univalents (fig. $7 a$ ) these have approximately the same distance from the centrosome as the $\mathrm{X}$ group and are also lying 
outside the spindle. The $\mathrm{X}$ group, though it has two centromeres, behaves as if it were a single univalent. The absence of repulsion between these centromeres is conspicuous.

\section{(iv) The origin of the $X_{1} X_{2} O$-mechanism}

The cytological evidence does not give any indication of homology between the X's. But this, of course, is not conclusive. Even the difference in length which can be seen in Aranea (plate, fig. 6, at $\mathrm{X}_{m}$ ) and, more distinctly, in Tegenaria, does not exclude a partial homology.

As Painter (I9I4) has already pointed out the $\mathrm{X}_{1} \mathrm{X}_{2} \mathrm{O}$-mechanism in spiders must be very old. From a purely cytological viewpoint it might have originated from an XO-system by duplication of the $\mathrm{X}$. Four homologous X's in the female would not necessarily lead to frequent non-disjunction as strong interference, limiting the chiasma frequency to one per chromosome, might easily have prevented the formation of trivalents and quadrivalents. The chiasma frequency seems to be very low in all spiders.

The assumption of a duplicated XO-system leads, however, to most serious difficulties in regard to sex determination. Diplogenotypic sex determination with heterozygous males is based on a quantitative relation between the valency of F-genes in the X and of M-factors in the autosomes and possibly in the cytoplasm. To get fertile males and females, both with their normal sex chromosome number duplicated, would require a genetical change from $\mathbf{F}$ and $\mathbf{M}$ to, say, $\overline{\mathrm{F}}$ and $\overline{\mathrm{M}}$ with the new valency proportion $(\overline{\mathrm{M}}):(\overline{\mathrm{F}})=2(\mathrm{M}):(\mathrm{F})$. This change might have taken place by one mutation or, much more likely, by gradual development of races with different sexual strength so that $\bar{F}$ from one race and $\bar{M}$ from another would give the doubled valency proportion. But in both cases I fail to see how $\bar{M}$-autosomes or $\overline{\mathrm{M}}$-cytoplasm and 2, respectively $4 \overline{\mathrm{F}}$-X's could be combined without first leading to individuals with abnormal valency proportion, sterile intersexes or supersexes.

The idea of duplication can be dismissed the more easily as another interpretation does not involve any serious difficulties. Centric fragmentation, as discussed by Darlington (1940), of one large X would not disturb sex determination if positive heterochromasy of the $\mathrm{X}$ did already exist in the prophase of the male. Then joint $\mathrm{X}$ segregation would be secured from the very beginning. In heterozygous females convergent $\frac{\mathrm{X}}{\mathrm{X}_{1} \mathrm{X}_{2}}$-trivalents would be formed in a fair majority of cases, thus keeping the frequency of non-disjunction low enough to permit an initial random spreading of the $X_{1} X_{2}$-system.

But a certain percentage of $\mathrm{X}_{1} \mathrm{XX}_{2}$-trivalents would probably reach linear co-orientation leading to eggs with abnormal sex complements. Therefore, once a population homozygous for the $\mathrm{X}_{1} \mathrm{X}_{2}$-system had been established, interbreeding with populations 
still containing the large $\mathrm{X}$ would be disfavoured by selection. So the $\mathrm{X}_{1} \mathrm{X}_{2}$-system may have helped to promote discontinuity within and finally the breaking up of the species.

Presumably the new $\mathrm{X}_{1} \mathrm{X}_{2}$-system had still another more immediate selective advantage. In most organisms the length differences within the chromosome set are comparatively small. Uniformity must be favoured by selection, apparently because it facilitates co-ordination between chromosomes. If the length of the $\mathrm{X}$ in the ancestors of spiders had become excessive, as is frequently the case for instance in Orthoptera, fragmentation would have increased length uniformity which, in fact, is rather conspicuous in spiders.

These views on the origin of the $\mathrm{X}_{1} \mathrm{X}_{2} \mathrm{O}$-mechanism are strongly supported by the conditions in Tegenaria (Revell, 1947). T. derhamii and $T$. domestica have an $\mathrm{X}_{1} \mathrm{X}_{2} \mathrm{X}_{3} \mathrm{O}$-mechanism, whilst $T$. atrica has only two X's as have all other spiders so far investigated ( 15 species, inclusive Aranea Reaumuri, from I5 genera). No doubt, T. atrica represents the original type. Now in this species the X's are considerably longer than the autosomes, and the larger $\mathrm{X}$ is about equal in length to the sum of the two smaller X's in T. domestica (Revell's plate, fig. 6) which have approximately the same length as the autosomes. Here the assumption of fragmentation is backed by direct evidence. Obviously an event which has happened in an ancestor of all present spiders has been repeated within the genus Tegenaria.

It must be noted that in Tegenaria as apparently in all spiders the $X$ 's have (sub)terminal centromeres. Therefore, two X's of about equal length cannot have derived from one simply by centric fragmentation. Let us assume that misdivision of the centromere was combined with a break near the end of the long arm to produce a long inversion with subsequent breakage of the resulting dicentric chromosome. This conclusion is more satisfying anyhow than mere centric fragmentation as it implies only the healing of a free chromatid end, an event already observed (McClintock, I939), and not that of a misdivided centromere in end position which has so far proved to be unstable (Darlington, I940).

The origin of a multiple XO-mechanism is cytologically quite different from that of the $\mathrm{X}_{1} \mathrm{X}_{2} \mathrm{Y}$-system in the sub-family Mantinæ of the Mantoidea. White (1940b, I94I) has clearly shown that this developed from an XO-system by translocation between the $\mathrm{X}$ and one autosome. In regard to sex determination, however, both ways meant the same : a breaking up of one carrier of F-genes in two not involving any change in the quantity of these genes which retain their joint segregation in the male.

\section{MEASUREMENTS OF CHROMOSOME LENGTH}

The length $l_{p}$ of the chromosome projection was taken from drawings; height-differences $h$ were read from the fine adjustment scale. The methods for the calculation of the true length $l$ are described 
in the legend of table 2 in which the results are comprehended. A list of previous chromosome measurements, including some of those presented here, has been published by Darlington and Upcott (I939).

TABLE 2

Average length of single chromosomes

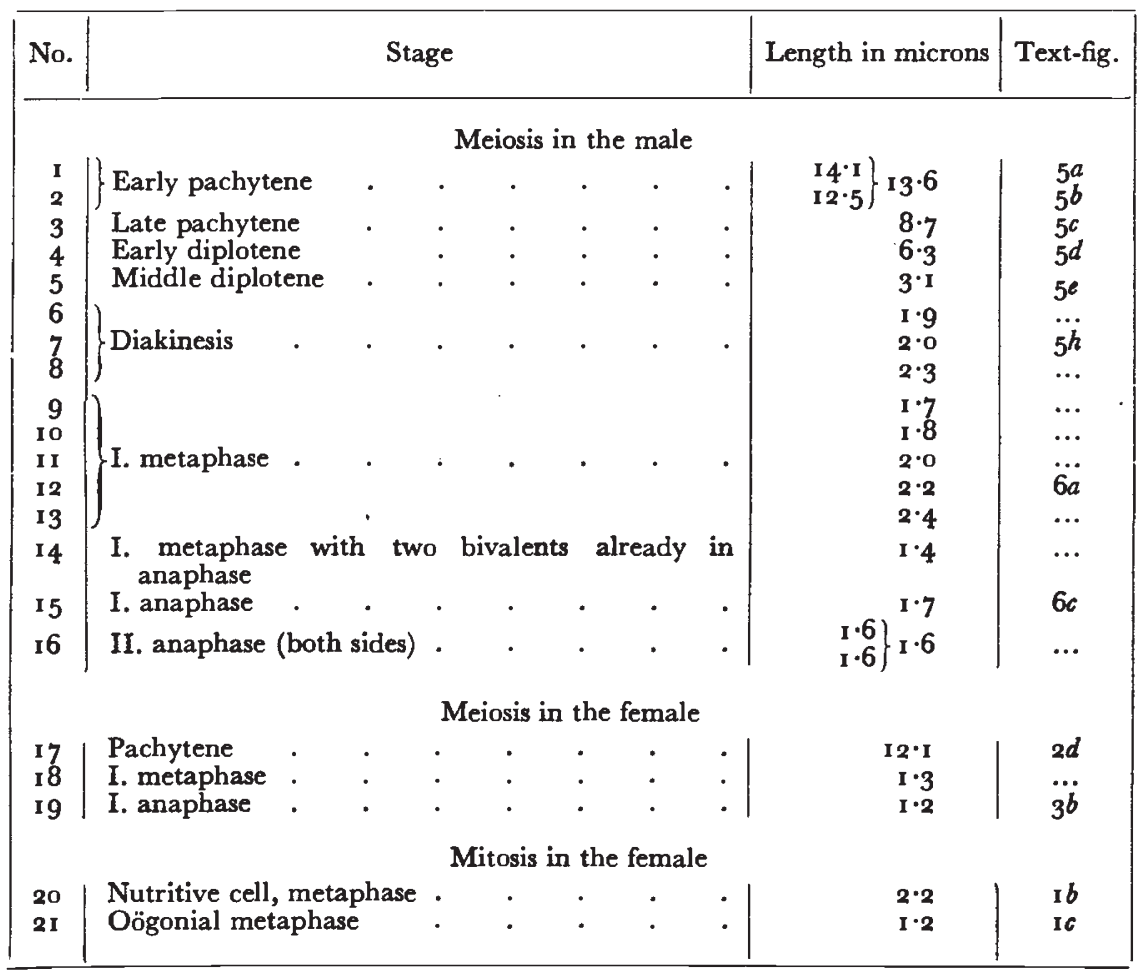

Notes on table 2

In nos. 9-16 and $18-21$ the true length $l$ was either equalled to the length $l_{p}$ of the projection or calculated from $l_{p}$ and the height difference $h$, if the chromosome was not lying sufficiently parallel to the plane of projection. In nos. $1-8$ and 17 the chromosomes showed no strong preferential orientation and the formula $1=\frac{4}{\pi} l_{\text {p }}$ (Pätau, 1937b) could be used. In no. 1 the axis of polarisation formed nearly a right angle with the optical axis while in no. 2 both were approximately parallel. Therefore $l$, as obtained by the formula, must be too large in no. I and too small in no. 2. From geometrical reasons the weighted mean $\frac{2.14 \cdot 1+12 \cdot 5}{3}=13 \cdot 6$ has to be taken as best estimation of the true value. In no. 15 those chromosomes were selected which were practically parallel to the plane of projection. In the other nuclei all chromosomes were measured, either inclusive (nos. 17-21) or without the X's. Since the length of each $\mathbf{X}$ cannot be very different from the average length of the autosomes, the error thus introduced must be quite small.

In Aranea the following points are of interest :-

(I) During pachytene a considerable shortening takes place.

(2) The original pachytene length in male and female is about the same. By comparison with a high number of other nuclei, nos. I 
and 2 (table 3 ) could be identified as early pachytene but this cannot be said in regard to the female nucleus no. 17. Therefore the fact that the chromosomes in this nucleus appear to be shorter than in the early pachytene of the male does not prove a difference in the intial pachytene length between male and female. So the value $13.6 \mu$ from nos. I and 2 can be regarded as the best available approximation to the original chromonema length. Table 3 has been based on it.

\section{TABLE 3}

\section{Shortening factor relative to chromonema length $13 \cdot 6 \mu$} as measured in pachytene of male

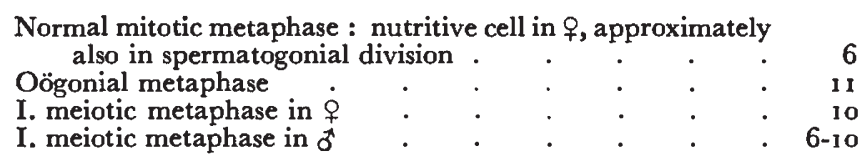

Note--The spiralisation coefficient (factor of maximum contraction in meiosis, Darlington and Upcott, 1939) is about 1o in both sexes.

(3) Two types of mitotic chromosomes were observed (table 3 ). Those in oögonial metaphase had only half the length of that found in the divisions of nutritive cells in the female. The chromosomes of the last spermatogonial division were not measured but the comparison of the figs. I $b$ and $4 a$ shows clearly enough that they have practically the same length as the nutritive cell chromosomes of the female.

(4) The chromosomes of the first meiotic division in the egg are as short as those of the oögonial mitosis.

(5) In the first meiotic metaphase of the male the chromosomes of the same cyst have the same length but the variation between cysts covers nearly the whole range between long and short mitotic chromosomes. Great differences in chromosome length occur even between cysts which are not very far apart in the testis (plate, fig. 6 at $s$ and $l$ ). This variation is already visible in diakinesis and cannot be due to shortening during metaphase. It must be caused by intercystic variation of conditions determining spiralisation which probably depend on the location of the cysts within the testis. No conspicuous length variation was found in the eggs. But these have an environment of their own, strongly protected against external influences by the egg membrane.

In regard to spiralisation the following conclusions can be drawn. The length of metaphase chromosomes is at least six times that of pachytene chromosomes and can therefore not be accounted for by a mere compact spiral of a cylindrical chromonema unless we assume a shortening of the chromonema itself by a factor of about $2 \cdot 2$. The length decrease during pachytene may be caused by this shortening and does therefore not prove that the external coiling of the chromonema has already started. The considerable difference between the two types of mitotic chromosomes (fig. I $b$ and $c$ ) strongly suggests that the oögonial, like the short meiotic chromosomes, have a double 
system of coiling. An indication of major coils can be seen in the dicentric chromosome of fig. $7 d$.

The length variation in diakinesis and in the first metaphase of the male must be due to varying degrees of spiralisation, but it would be rash to assume that the bivalents of the longest type have only minor coils. In Tradescantia, as will be shown elsewhere, the thread of the major coil in first metaphase is much longer than the mitotic metaphase chromosome with its single spiral. With a lower but still noticeable degree of coiling in the major spiral the meiotic chromatid could attain the length of a singly-coiled chromatid. That may be the case in Aranea. It seems the more probable as it would explain the length variability. A double spiral ought to be less stable than a single spiral.

\section{SUMMARY}

The diploid number of autosomes is 22. The male has two X's which pass always to the same pole in the first division. The female has four X's which cannot be distinguished from the autosomes and which form bivalents as these do.

Heterochromasy is displayed only by the X's and only in spermatogenesis and in $\mathrm{X}_{1} \mathrm{X}_{2}$-spermatids.

The joint $\mathrm{X}$-segregation at meiosis is caused by heterochromatic stickiness which keeps the X's together until, with the beginning of metaphase, they are connected with one centrosome.

It must be assumed that, within the strongly condensed X's of the meiotic prophase in the male, the same coiling process takes place as in the stretched autosomes.

A nucleic-acid-containing fluid, different from the nucleic acid attached to the chromonema, would account for the smooth outline of chromosomes from late prophase to anaphase, for the heterochromatic over-condensation in prophase and resting nuclei, and for the heterochromatic stickiness. This matrix would not influence spiralisation.

A new argument in favour of a compact spiral structure in metaphase is advanced from the constancy of length with varying nucleic acid charge.

The chromosome length at different stages was measured and interpreted in terms of spiral structure. The initial pachytene length in male and female is not very different if at all.

It is highly probable that the two X's in spiders have never been homologous but have arisen from one large $\mathrm{X}$ by fragmentation caused directly or indirectly by misdivision of the centromere. This event has been repeated within the genus Tegenaria, thus leading to species with three X's.

\section{REFERENCES}

CASPERSSON, T., AND SCHULTZ, J. 1938.

Nucleic acid metabolism of the chromosomes in relation to gene reproduction.

Nature, Lond. I42, 294-5. 
DARLINGTON, c. D. 1936 .

Crossing-over and its mechanical relationships in Chorthippus and Stauroderus.

7. Genet. 33, 467-500.

DARLINGTON, C. D. 1937 .

Recent advances in Cytology, 2nd ed. London : Churchill.

DARLINGTON, C. D. 1939 .

The genetical and mechanical properties of the sex chromosomes. V. Cimex and the Heteroptera.

7. Genet. 39, $101-137$.

DARLINGTON, c. D. 1940 .

The origin of iso-chromosomes.

7. Genet. 39, 35 I-6 I.

DARLINGTON, C. D. $\quad$ I 947 .

Nucleic acid and the chromosomes.

Symp. Soc. Exp. Biol. I, Nucleic acid, pp. 252-269.

DARLINGTON, c. D., AND LA COUR, L. F. 1938.

Differential reactivity of the chromosomes.

Ann. Bot., Lond., N.S. 2, 615-25.

DARLINGTON, C. D., AND LA COUR, L. F. 1940.

Nucleic acid starvation of chromosomes in Trillium.

7. Genet. 4o, 185-213.

DARLINGTON, C. D., AND LA COUR, L. F. 1945.

Chromosome breakage and the nucleic acid cycle.

7. Genet. $46,180-267$.

DARLINGTON, C. D., AND UPCOTT, M. B. 1939.

The measurement of packing and contraction in chromosomes.

Chromosoma I, 23-32.

HARD, W. L. $\quad$ I939.

The spermatogenesis of the lycosid spider, Schizocosa crassipes (Walckenaer).

7. Morph. 65 , 121-154.

McClintock, B. 1939.

The behaviour in successive nuclear divisions of a chromosome broken at meiosis.

P.N.A.S. 25, 405-416.

MONTGOMERY, T. H. 1907 .

On the maturation mitoses and fertilisation of the egg of Theridium.

Zool. Jahrb., Abt. Anat. u. Ont. 25, 237-250.

PAINTER, T. S. I9I4.

Spermatogenesis in spiders.

Zool. Jahrb., Abt. Anat. u. Ont. 38, 509-576.

PÄTAU, K. $1937 a$.

Partiell heterochromatische Chromosomen bei Radiolarien.

Arch. Protistenk. 89, 393-404.

PÄTAU, K. $1937 b$.

SAT-Chromosom und Spiralstruktur der Chromosomen der extrakapsulären Körper (Merodinium spec.) von Collozoum inerme Müller.

Cytologia, Fujii-Jubiläumsbd., 667-68o. 
REVELL, S. H. 1947.

Controlled X-segregation in Tegenaria.

Heredity $x, 337-347$.

SCHRADER, F. $194 \mathrm{I}$.

Heteropycnosis and non-homologous association of chromosomes in Edessa irrorata (Hemiptera Heteroptera).

7. Morph. 69, 587-604.

THOMAS, P. T., AND REVELL, S. H. 1946.

Secondary association and heterochromatic attraction. I. Cicer arietinum.

Ann. Bot. 9, 159-164.

WHITE, M. J. D. 1935 .

The effects of $\mathrm{X}$-rays on mitosis in the spermatogonial divisions in Locusta migratoria $\mathrm{L}$. P.R.S. B, $119,6 \mathrm{I}-84$.

WHITE, M. J. D. I $940 a$.

The heteropycnosis of sex chromosomes and its interpretation in terms of spiral structure.

7. Genet. 40,67-82.

WHITE, M. J. D. $1940 b$.

The origin and evolution of multiple sex-chromosome mechanisms.

7. Genet. 40, 303-336.

WHITE, M. J. D. I94I.

The evolution of the sex chromosomes. I. The $\mathrm{XO}$ and $\mathrm{X}_{1} \mathrm{X}_{2} \mathrm{Y}$ mechanism in praying mantids.

F. Genet. 42, 143-1 72. 